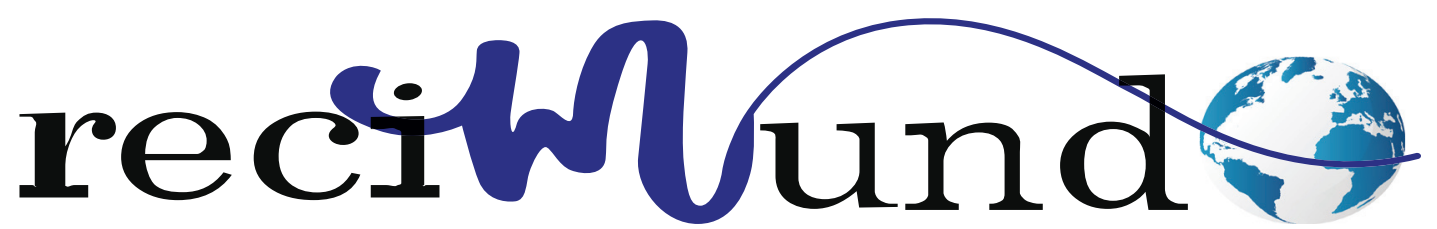

Revista Científica Mundo de la Investigación y el Conocimiento

DOI: 10.26820/recimundo/4.(4).octubre.2020.182-191

URL: http://recimundo.com/index.php/es/article/view/890

EDITORIAL: Saberes del Conocimiento

REVISTA: RECIMUNDO

ISSN: 2588-073X

TIPO DE INVESTIGACIÓN: Reporte de caso

Código UNESCO: 32 Ciencias Médicas

PAGINAS: 182-191

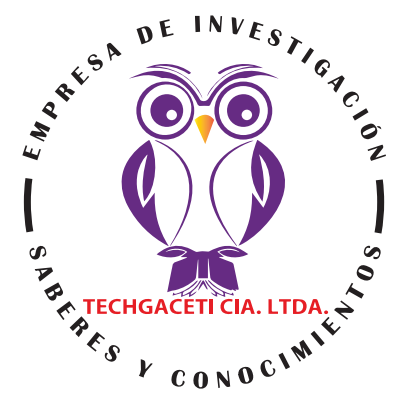

\title{
Hemorragia Subaracnoidea Aneurisma Cerebral. Presentación de Caso Clínico
}

Subarachnoid Hemorrhage Cerebral Aneurysm. Presentation of Clinical Case Hemorragia Subaracnoidea Aneurisma Cerebral. Presentación de Caso Clínico Néstor Ricardo Espinosa Sánchez'; Fanny Alcira Reyes Neira²; Yustin Antonieta Torres Yamunaque³; Karen Lisseth Cacay Ramos ${ }^{4}$

RECIBIDO: 10/07/2020 ACEPTADO: 26/08/2020 PUBLICADO: 15/10/2020

1. Médico de la Universidad de Guayaquil; Investigador Independiente; Guayaquil, Ecuador; drnespinosa@hotmail.com; https://orcid.org/0000-0002-5207-4189

2. Médico de la Universidad Particular de Especialidades Espíritu Santo; Investigador Independiente; Guayaquil, Ecuador; fanita_reyes_15@hotmail.com; (D) https://orcid.org/0000-0002-6924-544X

3. Médico de la Universidad de Guayaquil; Ministerio de Salud Pública; Guayaquil, Ecuador; justhinetorres@gmail.com; https://orcid.org/0000-0001-9093-7451

4. Médico de la Universidad Técnica de Machala; Investigador Independiente; Guayaquil, Ecuador; karen.cacay@gmail.com; (iD) https://orcid.org/0000-0002-5706-8845

CORRESPONDENCIA

Néstor Ricardo Espinosa Sánchez

drnespinosa@hotmail.com

Guayaquil; Ecuador

ㄷ RECIMUNDO; Editorial Saberes del Conocimiento, 2020 


\section{RESUMEN}

Se define como una colección de sangre dentro del parénquima cerebral producida por una rotura vascular no traumática. Se reporta paciente masculino de 61 años de edad quien acude a centro hospitalario por presentar cefalea holocraneana de gran intensidad, descrita como la peor de su vida a la cual se le suministraron analgésico intravenoso y fue dado de alta. Quien posterior a 3 días presenta sincope mas cefalea de mayor intensidad con vomito en escopetazo, picos hipertensivos por lo cual acude nuevamente a esta casa de salud donde realizan TAC de cráneo donde reporta hemorragia subaracnoidea FISHER II HH II. Se solicita interconsulta con neurocirugía el cual acude al momento y programa cirugía de emergencia para embolizacion de aneurisma sacular roto de arteria cerebral anterior izquierda más aneurisma en arteria cerebral anterior derecha. Posterior a 21 días de hospitalización en UCl y 7 días en hospitalización es dado de alta con buen estado neurológico sin déficit motor ni perdida de sensibilidad en extremidades.

Palabras clave: Hemorragia Subaranoidea (HSA), Aneurisma Intracraneales múltiples (AIM), Embolizacion de Aneurisma.

\section{ABSTRACT}

Is defined as a collection of blood within the cerebral parenchyma produced by a non traumatic vascular rupture. A 61 year old male patient is reported who comes ti the hospital due to sereveholocranial headache, described as the worst in his life, who was given intravenous analgesic and was discharged. Who after 3 days presents syncope plus headache of greater intensity with vomiting is shotgun, hypertensive peaks for which he goes back to the health home where they result in a CT scan of the skull where he report subarachnoid hemorrhage Fisher II HH II. Consultation with neurosurgery is requested who attends at the moment and schedules emergency surgery for embolization of sacular aneurysm in left anterior cerebral artery plus aneurysm in right anterior cerebral artery. After 21 days of hospitalization in the ICU and 7 days of hospitalization, he was discharged with good neurological status without motor deficit or loss of sensation in the ectremities.

Keywords: Subararachnoid (SAH), Multiple Intracraneal Aneurysms (MIA), Aneurysm Embolization.

\section{RESUMO}

É definido como uma coleção de sangue dentro do parênquima cerebral produzida por uma ruptura vascular não traumática. Há relato de paciente do sexo masculino, 61 anos, que chega ao hospital por apresentar cefaléia soro-holocraniana, descrita como a pior de sua vida, que recebeu analgésico intravenoso e teve alta. Quem após 3 dias apresenta síncope mais cefaléia de maior intensidade com vômitos é espingarda, picos hipertensivos pelos quais volta ao sanatório onde resultam em tomografia computadorizada de crânio onde relata hemorragia subaracnóidea Fisher II HH II. Solicita-se consulta com neurocirurgia quem comparece no momento e agenda cirurgia de emergência para embolização de aneurisma sacular em artéria cerebral anterior esquerda mais aneurisma em artéria cerebral anterior direita. Após 21 dias de internação na UTI e 7 dias de internação, recebeu alta em bom estado neurológico, sem déficit motor ou perda de sensibilidade nas extremidades.

Palavras-chave: Subaracnoide (SAH), múltiplos aneurismas intracranianos (MIA), embolização de aneurisma. 


\section{Introducción}

\section{Hemorragia Subaranoideo}

La hemorragia subaracnoidea (HSA) es cuarto trastorno vascular cerebral más frecuente de la aterotrombosis, la embolia y la hemorragia intracraneal primaria (hipertensiva). El concepto referente a que el aneurisma se debe a una dilatación de una arteria que presenta una debilidad en su estructura, se le atribuye a Lancisi en 1728. Morgagnia de Papua, al parecer, fue el primero en describir un aneurisma intracraneal en 1961. (García Zambrano, Sempértegui Rojas, \& Féliz Naveda, 2007)

Se define como una colección de sangre dentro del parénquima cerebral producida por una rotura vascular no traumática, puede abrirse al sistema ventricular o al espacio subaranoideo. El 85\% de los aneurismas intracraneales se encuentran en la circulación anterior y el 15\% restante en la circulación posterior. Se considera que el $30 \%$ de los aneurismas son múltiples. Entre los factores de riesgo para la formación de los aneurismas están: edad, sexo, raza, tabaquismo, alcoholismo, ingesta de anticonceptivos orales, embarazo, radioterapia por tumor cerebral, consumo de cocaína y anfetaminas. También se relacionan con ciertas enfermedades tales como: Hipertensión Arterial (HTA), Lupus Eritematoso sistémico, arteriosclerosis, síndrome de marfan, anemia de células falciformes, endocarditis infecciosa. (García Zambrano, Sempértegui Rojas, \& Féliz Naveda, 2007)

Puede ser primaria (70\%). Relacionada con la HTA o con angiopatía amiloide. Más frecuente en pacientes mayores, sexo femenino y de arterial crónica produce alteraciones degenerativas en la pared arterial. La angiopatía amiloide es la causa más frecuente de hemorragia lobar y superficial en el adulto mayor. Se produce una degeneración en la pared de las arterias y arteriolas situadas en las leptomeningues y en la cor- teza cerebral. Pueden producirse lesiones múltiples, recidivantes y más frecuentes en regiones posteriores.

La hemorragia secundaria es menos frecuente. Se presenta en pacientes más jóvenes. La localización preferente es lobar y está en relación con fármacos antiagregantes $\mathrm{O}$ anticoagulantes, la formaciones vasculares (MAV o cavernomas), coagulopatias o abuso de drogas (García de Sola, 2020).

Escala de Graduación de la HSA según la WorldFederation of Neurosurgical (WFNS): Consiste en una clasificación de los pacientes según la severidad de la presentación clínica. Ha demostrado una asociación directa con la morbi mortalidad. Si bien existen otras similares, algunas ampliamente difundidas y empleadas, como Hunt y Hess, se recomienda la WFNS sobre otras debido a; una buena correlación interobservador, atribuible a la utilización de una terminología más objetiva; una adecuada correlación entre mayor grado y peor pronóstico; amplia utilización a nivel mundial e incorporación en el sistema Vasogrande. (Tabla I).

\begin{tabular}{|l|}
\hline Escala de graduación de la HSA (según la WFNS) \\
\hline Grado 0: Glasgow 15; $\sin$ HSA \\
\hline Grado I: Glasgow 15; $\sin$ déficit focal \\
\hline Grado II : Glasgow 13-14; sin déficit focal \\
\hline Grado III: Glasgow 13-14; con déficit focal \\
\hline Grado IV: Glasgow 7-12; con o sin déficit focal \\
\hline Grado V: Glasgow 3-6; con o sin déficit focal \\
\hline
\end{tabular}

Tabla 1. Escala de Graduación de HSA

Fuente: (Huidobro Salazar \& Quintana Marín, 2017)

Escala de Fisher modificada (EFM): Tal como sucede con la clasificación de la severidad clínica, son múltiples las escalas que se han propuesto para estimar la probabilidad de ocurrencia de DCl. Muchas de ellas, se basan en una conocida asociación directa entre distribución/ cantidad de sangre y riesgo de vasoespasmo. (Tabla 2) (Huidobro Salazar \& Quintana Marín, 2017) 


\begin{tabular}{|l|}
\hline Escala de Fisher modificada \\
\hline Grados \\
\hline 0 - No HSA o hemorragia intraventricular \\
\hline 1 - HSA focal o difusa fina, sin hemorragia intraventricular \\
\hline 2 - HSA focal o difusa fina, con hemorragia intraventricular \\
\hline 3 - HSA focal o difusa gruesa, sin hemorragia intraventricular \\
\hline 4 - HSA focal o difusa gruesa, con hemorragia intraventricular \\
\hline
\end{tabular}

Tabla 2. Escala de Graduación de Fisher modificada

Fuente: (Huidobro Salazar \& Quintana Marín, 2017)

- ASPECTS Score es un sistema de puntuación de 10 puntos para cuantificar los cambios isquémicos tempranos en el territorio de la arteria cerebral media, con una puntuación de 10 que indica normal y 1 punto restado por cada región anormal.

- Una puntuación de ASPECTS menor o igual a 7 predice un peor resultado funcional a los 3 meses, así como una hemorragia sintomática.

- Según el estudio realizado por RI Aviv et al., Los pacientes con ASPECTOS puntuaron menos de 8 tratados con trombólisis no tuvieron un buen resultado clínico ${ }^{3}$

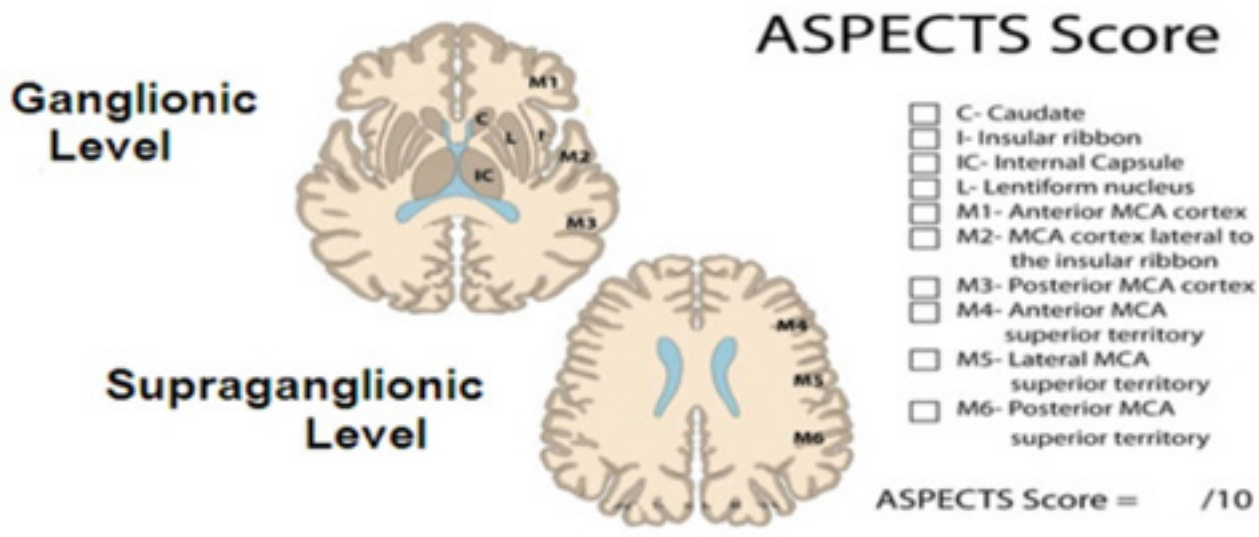

\section{8-10 small core 6-7 moderate core $\quad$ 0-5 large core}

Figura 1. Aspects Score

Fuente: (Huidobro Salazar \& Quintana Marín, 2017)

\section{Manifestaciones clínicas y sintomatológi- ca diagnostica}

La forma mas común de la HSA es la cefalea. Se trata de usualmente de una cefalea intensa de comienzo súbito, que alcanza su acné en segundos y minutos. En un tercio de los casos es la única manifestación, y son aquellos en los que el diagnostico puede pasarse por alto. Puede asociarse síntomas como perdida de la conciencia, que predice la hemorragia aneurismática, nauseas o vómitos, focalidad neurológica o crisis comiciales. (Vivancos, y otros, 2012).

\section{Presentación del Caso}

\section{Caso Clínico}

Paciente masculino de 61 años de edad.

Antecedentes Personales:

- Portador de marcapaso por bloqueo auriculoventricular en 2019.

- Hipertensión Arterial tratado con Losartan (suspendido hace 1 mes por medico tratante).

- Niega alergias. 
- Quirúrgicos: Colocación de marcapaso hace 1 año, colecistectomía, apendicectomizado hace 30 años, reparación del tendón de Aquiles.

- Hábitos: enolicos todos los fines de semana hasta llegar a la embriaguez. Tabáquicos: niega.

- Ocupación: Guardia de seguridad.

\section{Motivo de Consulta}

Cefalea con deterioro de sensorio

Se trata de paciente masculino de 61 años de edad quien es referido desde IESS de Guaranda, traído a esta casa de salud en ambulancia sin soporte de oxigeno con Glasgow 15/15 donde permaneció hospitalizado por 3 días por presentar cuadro clínico de 3 días de evolución previo a su ingreso a dicha unidad caracterizado por cefalea holocraneana de gran intensidad, descrita como la peor de su vida a la cual se le suministraron analgésico intravenosos y fue dado de alta.

Posterior a esos 3 días el paciente presenta sincope mas cefalea de mayor intensidad con vomito en escopetazo, picos hipertensivos por lo cual acude nuevamente a esta casa de salud donde realizan TAC DE cráneo donde reporta hemorragia subaracnoidea FISHER II HH II, por lo cual es derivado a la ciudad de Guayaquil con código de derivación.

Por lo cual acude nuevamente a esta casa de salud, llega a nuestra casa hospitalaria, hospitalaria Hospital Clínica San Francisco con diagnostico de ingreso a la unidad de cuidados intensivos hemorragia subaracnoidea FISHER III HUNTHESS II, con score sofá 0 puntos y APACHE 10 PTS. Por lo cual se realiza interconsultade emergencia al servicio de neurocirugía el cual acude al momento y programa cirugía de emergencia para embolizacion de aneurisma sacular roto de arteria cerebral anterior izquierda más aneurisma en arteria cerebral anterior derecha.
Posterior a la embolizacion presenta síndrome inadecuado de hormona anti diuréticaSIADH e INTERCURRE con accidente cerebro vascular isquémico en territorio de cerebral media como complicación del procedimiento realizado.

Días posteriores INTERCURRE con sepsis de foco a determinar que al la realización de exámenes complementarios se logra diagnosticar neumonía asociada a la ventilación mecánica. Por lo cual se colocaron dispositivos invasivos catéter venoso central yugular derecho el 24 de agosto del 2020 hasta el 29 de agosto del mismo año, sonda vesical del 24 de agosto hasta el 29 de agosto del mismo año, línea arterial radial derecha del 25 de agosto al 29 de agosto del mismo año.

Dentro de la antibiticoterapia se uso meropenem cuando intercurrio con neumonía mas vancomicina desde el 29 de agosto hasta el 3 de septiembre del mismo año.

Se realizaron cultivo los cuales en aspirado bronquial presenta Staphylococcus auerus. El 30 de agosto de 2020 en hemocultivos Staphylococcus auerus, en hisopado rectal sin desarrollo bacteriano. El dia 30 de agosto de 2020 en cultivo de orina sin desarrollo hasta el 30 de agosto de 2020. Una vez que se resuelve proceso respiratorio, paciente se logra extubar el 30 de agosto de 2020 y se logra dar pase a piso de hospitalización. En los días posteriores paciente se sigue el caso estudiándolo donde sintomatología y por medio de la clínica se llega a la conclusión de que el paciente además de su patología actual presentaba enfermedad de EHLERS - DANLOS con riñones poliquisticos hereditaria.

\section{Comentarios}

\section{TAC Cráneo (24/8/2020)}

Hemorragia subaracnoidea difusa distribuida en surcos de la convexidad bilateral con 
discreto nivel hemático en astas occipitales de ventrículos laterales, llama la atención imagen nodular en cisterna cerebelosa superior, paramedial izquierda, inespecífica, se puede complementar con Resonancia Magnética (RM). Parénquima cerebral sin evidencia de lesiones ocupantes de espacio ni alteración de la relación sustancia gris - blanca, cisterno - ventricular conservado, estructuras de la línea media nos desplazadas.

\section{Angiotac de Cráneo}

El estudio practicado demuestra aneurisma sacular en segmento M2 de la arteria cerebral media derecha de mide $4 \times 3 \mathrm{~mm}$, cuello 2,5 $\mathrm{mm}$, aneurisma sacular en segmento A2 de arteria cerebral anterior izquierda que mide $5 \times 4 \mathrm{~mm}$, cuello de $2 \mathrm{~m}$, dos aneurismas saculares en segmento A3 de arteria cerebral anterios derecha miden $4 \times 2,5 \mathrm{~mm}$ respectivamente, senos venoso sin alteraciones.

\section{Procedimiento (24/8/2020}

Embolizacion de 3 aneurismas del territorio de la arteria cerebral anterior (ACA) (Aneurisma pericalloso, aneurisma calloso marginal derecho, aneurisma del segmento A2 derecho).

\section{Examen Físico}

\section{Neurológico}

Consiente, apertura ocular espontanea con pupilas reactivas a la luz, Glasgow 15/15 sin focalidad neurológica, moviliza extremidades, sigue órdenes complejas.

\section{Cardiovascular}

Hemodinamicamente estable sin soporte vasopresor con TAM:70mmHg con FC:69 con RSCS rítmicos sin soplos, sin tercer ruido, sin agregados con buena perfusión distal y buen llenado capilar de 3 seg.

\section{Respiratorio}

Con buena mecánica ventilatoria con canula de oxigeno a 3 litros, saturando 99\% con CSPS ventilados, murmullo vesicular conservado, sin agregados.

\section{Digestivos}

Abdomen blanco, depresible no doloroso, con ruido hidroaereos presentes, no presenta catarsis, sin masas palpables, sin viceromegalias.

\section{Renal / Metabólico}

Paciente con diuresis adecuada de 200cc en 24 horas con azoados en valores dentro de los parámetros normales con medio interno adecuado.

\section{Osteomuscular}

Extremidadessimétricas, sin edemas, ni ulcera de presión, sin escara, sin embargo intercurrio con deformidad de articulación de miembro superior a nivel del codo con fractura del mismo mas luxación.

\section{Infecciosos}

Afebril con marcadores infecciosos negativos.

Paciente posterior a 21 días de hospitalización en $\mathrm{UCl}$ y 7 días en hospitalización es dado de alta con buen estado neurológico sin déficit motor ni perdida de sensibilidad en ninguna extremidad, Hemodinamicamente estable con buena mecánica ventilatoria con aparato digestivo sin presentar anomalías con buena función renal y diuresis. 


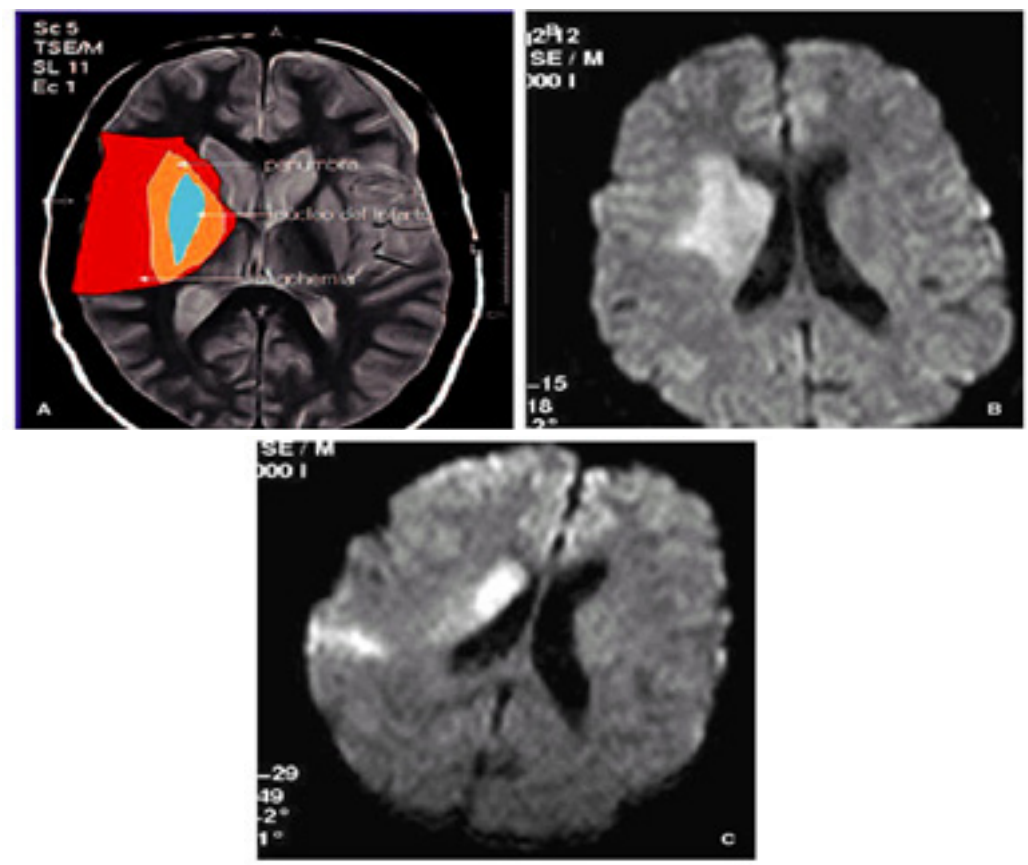

Figura 2. Resonancia Magnética. (RM)

Fuente: Los autores

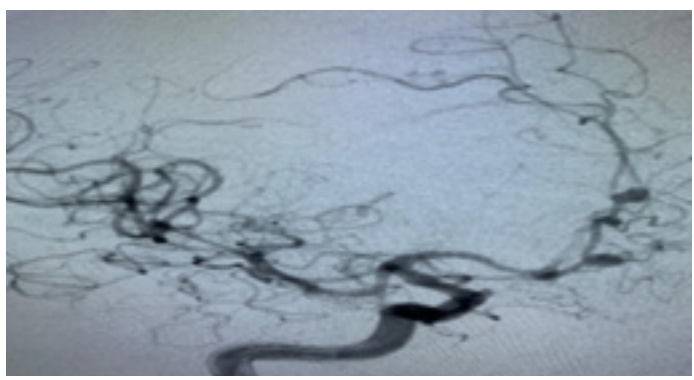

Figura 3. Foto Panangiografia Cerebral.

Fuente: Los autores

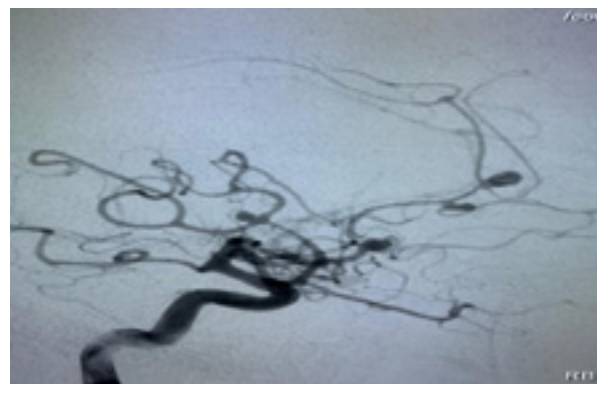

Figura 4. Foto Panangiografia Cerebral.

Fuente: Los autores 


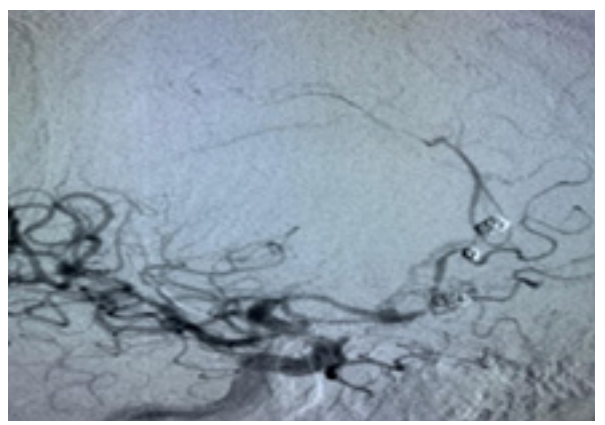

Figura 5. Foto Panangiografia Cerebral.

Fuente: Los autores

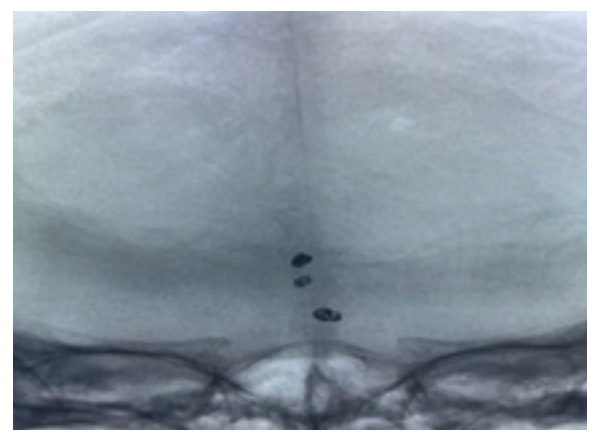

Figura 6. Foto Panangiografia Cerebral.

Fuente: Los autores

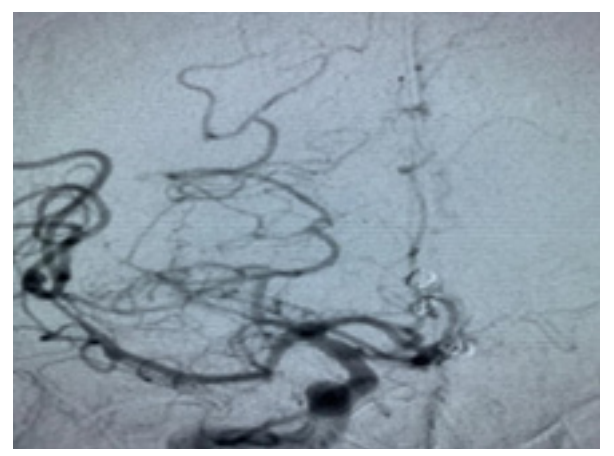

Figura 7. Foto Panangiografia Cerebral.

Fuente: Los autores 


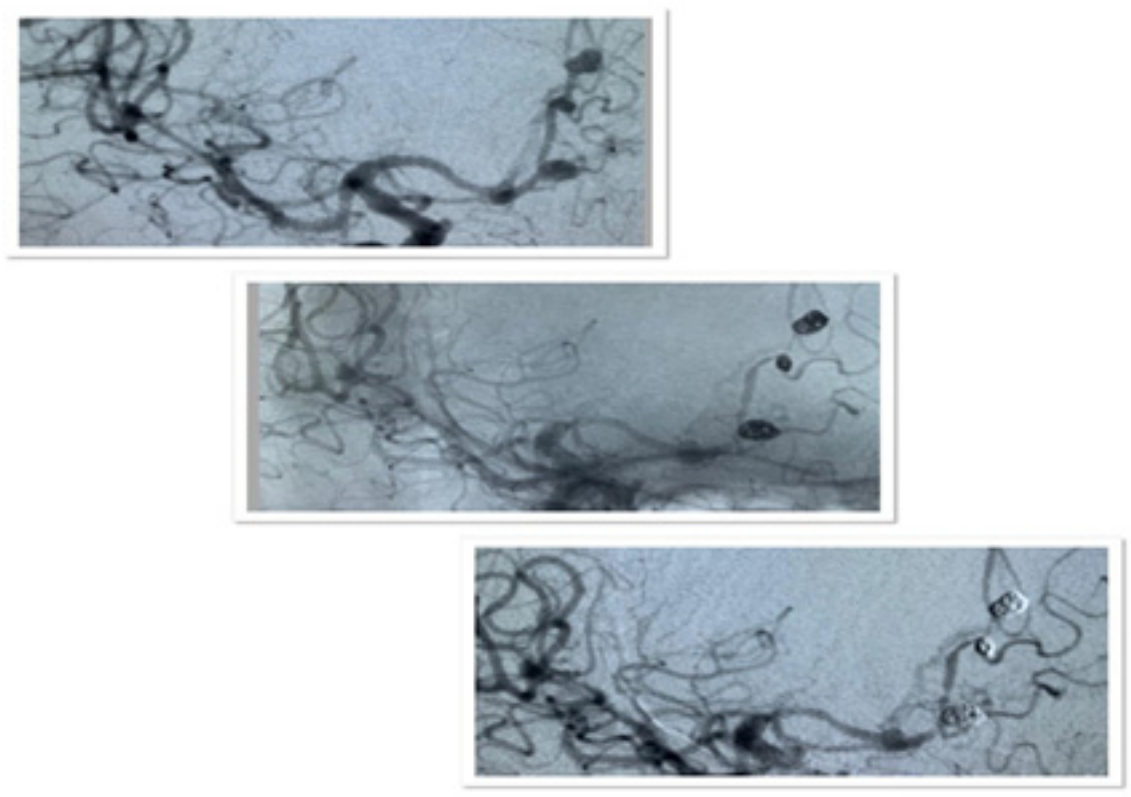

Figura 8. Foto Panangiografia Cerebral.

Fuente: Los autores

\section{Discusión}

La HSA, debido a la ruptura de un aneurisma, es un evento devastador asociado a tasas elevadas de morbi-mortalidad. (García Zambrano, Sempértegui Rojas, \& Féliz Naveda, 2007)

La alta morbi-mortalidad y mayor incidencia entre la $5^{\circ}$ y $6^{\circ}$ década de la vida, generan un alto costo asociado a esta patología, tanto económico, como social debido a la discapacidad que genera en un segmento productivo de la población. (Huidobro Salazar \& Quintana Marín, 2017)

El estudio de Omama et al.mostro una relación entre HSA y la hora de presentación, con una curva de incidencia bimodal, ya descrita previamente para los ictus hemorrágicos. Se postula que esta relación con el ritmo cicadino se explicaría por las variaciones de las cifras de presión arterial a lo largo del día, y con el incremento de la agregación plaquetería descrito durante el despertar (Vivancos, y otros, 2012)

Se considera que en $15 \%$ a $33 \%$ de los ca- sos de HSA, se ha encontrado más de un aneurisma determinándose que la HTA es el factor de riesgo más importante asociado a la multiciplicidad. (García de Sola, 2020). Es importante realizar un diagnostico oportuno así como diferencial para tratar a tiempo a los pacientes con HSA y así evitar que aumente la tasa de mortalidad.

Ellamushi H. y colaboradores, en Londres, realizaron un estudio retrospectivo de todos los pacientes que, con diagnostico de HSA, ingresaron al National of Neurology and Neurosurgery, entre 1985 y 1997. De un total de 392 pacientes (287 F, 105 M) cuyas edades oscilaban entre 20 y 54 años de edad, 284 tenían 1 aneurisma y 108 $(27,5 \%)$ representan múltiples aneurismas, 68 pacientes con 2 aneurismas, 22 con 3 aneurismas, 13 con 4 aneurismas y 5 con 5 aneurismas. Se encontraron como factores de riesgo: HTA, tabaquismo, sexo femenino, edad (posmenopáusica) e historia familiar de enfermedad cerebro vascular. (García Zambrano, Sempértegui Rojas, \& Féliz Naveda, 2007)

En el estudio español, hasta el $68 \%$ de los 
pacientes presenta buena situación clínica a su llegada, definida como grados I-III de la escala de la federación mundial de neurocirujanos que implica una puntuación en la escala de coma de Glasgow entre 13 y 15. Este dato es significativo a la decisión de hospitalización de los casos de HSA en las unidades de ictus. El 19\% se clasifico como HSA idiopáticas tras un estudio angiografico inicial negativo. De estas, un 40\% fueron HSA peri encefálicas y hasta el $10 \%$ el estudio de imagen fue normal. Los pacientes con HSA peri encefálica o con neuroimagen presentan recuperación completa a los 6 meses hasta en el 90\%. En el caso de las HSA aneurismáticas, la localización más frecuente del aneurisma es la arteria comunicante anterior (36\%), seguida de cerebral media (26\%), comunicante posterior (18\%) y carótida interna (10\%). Un 9\% de los casos son aneurismas de circulación posterior y aneurismas múltiples en un $20 \%$. La incidencia de malformaciones arteriovenosas (MAV) asociada al 2\% (Vivancos, y otros, 2012).

\section{Bibliografía}

Ajay, S. (2018). Emergency Radiology. Imaging of Acute Pathologies (Second ed.). Boston: Springer.

García de Sola, R. (2020). Docencia: Hemorragia cerebral. Tema V. Unidad de Neurocirugía RGS, 1-5.

García Zambrano, W., Sempértegui Rojas, C., \& Féliz Naveda, D. (2007). Aneurismas Intracraneales Múltiples. Presentación de un caso y revisión de la literatura. Revista Ecuatoriana de Neurología, 16(1), 1-5.

Giraldo, A., Ucros, E., Varón, F., \& Mugnier, J. (2015). Dolor torácico: Más allá de un síndrome coronario. Reporte de caso. Revista Médica Risaralda, 21(1), 68-72.

Huidobro Salazar, J., \& Quintana Marín, L. (2017). Guía Clínica para el manejo de la hemorragia subaracnoidea aneuritica- propuesta de actualización al Ministerio de Salud de Chile. Revista Chiena de neurocirugia, 43(1), 1-3. Retrieved from https://www.neurocirugiachile.org/pdfrevista/v43_ n2_2017/huidobro_p156_v43n2_2017.pdf

Vivancos, J., Gilo, F., Frutos, R., Maestre, J., García-Pastor, A., Quintana, F., \& Roda, J. (2012). Guía de actuación clínica en la hemorragia subaracnoidea. Sistemática diagnóstica y tratamiento. Neurología, 29(6), 353-370. doi: 10.1016/j. nrl.2012.07.009

\section{CITAR ESTE ARTICULO:}

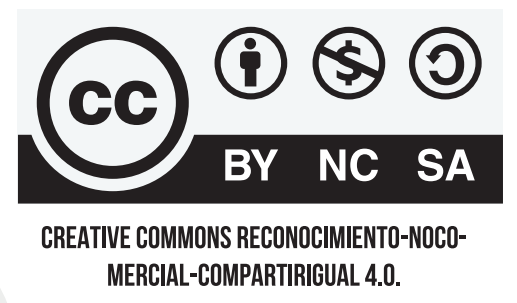

Espinosa Sánchez, N. R., Reyes Neira, F. A., Torres Yamunaque, Y. A., \& Cacay Ramos, K. L. (2020). Hemorragia Subaracnoidea Aneurisma Cerebral. Presentación de Caso Clínico. RECIMUNDO, 4(4), 182-191. https://doi.org/10.26820/ recimundo/4.(4).octubre.2020.182-191 